\title{
El periodismo slow digital de Jot Down y Gatopardo
}

\section{The digital slow journalism of Jot Down and Gatopardo}

\author{
Alba SABATÉ GAUXACHS' (DD 0000-0003-0956-9933 \\ Josep Lluís MICÓ SANZ1 (iD 0000-0003-1191-226X \\ Míriam DíEZ BOSCH' iD 0000-0002-3120-9443
}

\section{Resumen}

Descubrir el éxito de la combinación entre tradición e innovación. Este es el propósito del presente artículo, dedicado a analizar las claves del periodismo narrativo en el entorno digital a partir de los modelos de las revistas Jot Down y Gatopardo. Ante la inmediatez de la digitalización, los medios dedicados al periodismo narrativo rompen con las normas del espacio online y apuestan por el periodismo slow, basado en las técnicas tradicionales de los padres de este periodismo, como Tom Wolfe. Se estudian sus contenidos, técnicas, formatos y se examina cómo un periodismo centrado en la esencia del papel está disfrutando de una consolidada presencia y audiencia en el mundo digital. Al mismo tiempo, la propuesta es conocer la situación y reconocimiento de los periodistas en este tipo de medios; sus rutinas, procesos y condiciones laborales. Para ello se emplea una metodología basada en entrevistas en profundidad, análisis de contenido, observación no participante y revisión de la literatura. Las principales conclusiones muestran que, ante la situación actual del periodismo y de los medios de comunicación, priorizar la calidad a la inmediatez e hibridar la tradición con la innovación puede ser clave como garantía de futuro.

Palabras clave: Innovación. Periodismo. Periodismo digital. Periodismo literario. Tradición.

\begin{abstract}
To renew journalism without destroying tradition: this the goal of the article. It will analyze the main constituent elements of narrative journalism in the digital era using the models incorporated by the magazines Jot Down and Gatopardo. Challenged by the immediacy of the digital world, narrativejournalism seems/appears to break the digital rules. Media working on this kind of journalism place their bets on a slow method, based on traditional, tried-and-tested techniques, typified by authors such as Tom Wolfe and other professionals of narrative journalism. The article will study contents, formats, and techniques of news reporting that not only retain the essence of support papers, but also enjoy a consolidated audience in the digital world. It will be necessary to acquire a deep understanding of the situation in general, and the role of journalists in particular, in this landscape. This research is, above all, interested in their routines, processes and ways of working. The methodology used to examine this model is based on in-depth interviews, content analysis, non-participant observation and literature review. With all this in mind, and taking into account the current state of the media and the profession of journalism, the main conclusions show that this hybridization between innovation and tradition could be a successful formula, not just for survival but also as a way forward.
\end{abstract}

Keywords: Innovation. Journalism. Digital journalism. Literary journalism. Tradition.

\footnotetext{
$\overline{1}$ Universitat Ramon Llull, Facultat de Comunicació i Relacions Internacionals Blanquerna, Departamento de Periodismo. Plaça Joan Coromines, s/n., 08001, Barcelona, España. Correspondencia para/Correspondence to: A. SABATÉ GAUXACHS. E-mail: <albasg@blanquerna.url.edu>.

Apoio: Departamento de Empresa y Conocimiento de la Generalitat de Catalunya y Fondo Social Europeo.

Recibido el 16 del enero del 2018, versión final re-presentada el 17 del mayo del 2018 y aprobado el 6 del junio del 2018.
}

Como citar este artículo/How to cite this article

Sabaté Gauxachs, A.; Micó Sanz, J.L.; Díez Bosch, M. El periodismo slow digital de Jot Down y Gatopardo. Transinformação, v.30, n.3, p.299-313, 2018. http://dx.doi.org/10.1590/2318-08892018000300003 


\section{Introducción}

Los avances tecnológicos y la crisis económica han obligado al periodismo a reinventarse. El oficio ha vivido el lanzamiento de distintos modelos formales, de contenido y empresariales (Neveu, 2014). Han emergido medios que, con la filosofía y formato del periodismo literario (Bak; Reynolds, 2011), se desarrollan en el entorno digital. Ante la inmediatez, reporteo; ante la velocidad, calma; ante la brevedad, literatura (Boynton, 2012). Así se presenta el periodismo narrativo digital (Berning, 2011). Ajeno a la programación y calendarización, sigue el ritmo de las personas (Herrscher, 2012) y no el de la tecnología.

El periodismo narrativo es la especialidad que, con un método slow, explica historias reales con herramientas de la literatura (Le Masurier, 2015). No es el primero en dar la noticia, pero sí el único en explicarla en profundidad. El mundo iberoamericano ha vivido una emergencia de este tipo de medios durante la última década. El llamado big-bang (Albalad; Rodríguez, 2012) ha consolidado publicaciones en formato digital y papel.

Esta investigación estudia las claves de un periodismo que, rompiendo las normas digitales, demuestra que puede sostenerse bajo sus modelos empresariales. El objetivo es analizar rutinas y contenidos y examinar cómo adaptan el formato digital al papel y viceversa. La atención se focaliza en Jot Down y Gatopardo, modelos que hibridan tradición e innovación.

La muestra la configuran estos medios por varias similitudes que permiten trazar un hilo conductor, y diferencias que llevan a observar un marco de amplio alcance. Jot Down y Gatopardo tienen en común que trabajan en periodismo narrativo, en formato digital y en papel, y se sostienen económicamente. Además, comparten lengua y fidelidad al nuevo periodismo. Así, combinan elementos del periodismo digital con técnicas de la práctica tradicional de la profesión y de la literatura. La diferencia más clara es su origen. Ambas vienen de entornos distintos, teniendo en cuenta la localización y el soporte de nacimiento. Jot Down es española y surge como medio digital que posteriormente aparece en papel. Gatopardo nace en Colombia en papel y hace después el salto online. La revista colombiana surge en 2001; la española en 2011.

\section{La novedad de lo no tan nuevo}

Periodismo y literatura comparten objetivo: explicar una historia. La simbiosis entre ambos es el periodismo narrativo (Sims, 1996). Original de los sesenta en Estados Unidos (Wolfe, 1973), ha sido polémico desde su nacimiento, empezando por su denominación. Marsh (2010) habla de siete nombres para la narrativa de no ficción: Nuevo periodismo (Wolfe, 1973), novela de no ficción (Capote, 1965), relato corto de no ficción (Franklin, 1986), periodismo literario (Sims, 1990), periodismo narrativo (Franklin, 1996), periodismo de formato largo (Kirtz, 1998), periodismo literario narrativo (Hartsock, 2001) y nuevo nuevo periodismo (Boynton, 2005). Aunque todos presentan matices, según Albalad (2018) la denominación más presente en el mundo anglosajón es periodismo literario y la expresión es sinónima de la española periodismo narrativo (Gutiérrez Palacio, 2009; López Pan, 2010). Para Albert Chillón en una entrevista con Mejía (2017), el concepto periodismo narrativo es un pleonasmo. La International Association for Literary Journalism Studies (2006) acepta las dos acepciones en su Mission Statement. La controversia también se ha generado por el debate ético que levanta este periodismo al intentar trazar los límites entre reportaje y novela.

Chillón (1999), Bak y Reynolds (2011), Boynton (2012), Herrscher (2012), Angulo (2013) o Palau (2017) son autores que reflexionan sobre este periodismo. Chillón y Palau desde una perspectiva histórica y formal. Boynton (2012) y Bak (2011) con la visión estadounidense y tratando el contenido; Herrscher enfoca su análisis desde una óptica iberoamericana.

Wolfe (1973) es considerado por sus pares uno de los padres del nuevo periodismo. En El Nuevo Periodismo realiza un análisis teórico y es uno de los primeros en estudiar un periodismo que al mismo tiempo ejercía. Según Wolfe, las características del periodismo narrativo son: construcción escena por escena, diálogo, punto de vista en tercera persona y detallismo. Sims (1996) habla en los mismos términos de estructura, exactitud, vozy responsabilidad. 
Lo que Sims Ilama estructura se puede considerar la construcción escena por escena de Wolfe (1973); el objetivo, evitar que el texto sea cronológico. Ambos hablan de detallismo y exactitud en las descripciones de personajes y ambientes. Esta característica, según Herrscher (2012), es una herencia de la novela realista y de su evolución al naturalismo y al positivismo de Zola o Balzac. El periodismo de observación de Dostoievski o Chéjov es otro afluente del periodismo narrativo (Herrscher, 2012); también el teatro de Shakespeare. Esta es la voz de la que habla Sims (1996) y el punto de vista de Wolfe (1973); tanto en tercera como en primera persona, se trata de conseguir que el lector empatice. Así, el narrador omnisciente abunda en periodismo narrativo. Al usar la primera persona, el periodista marca su presencia. Ejemplos en esta práctica son Hemingway o Fallaci.

El concepto de diálogo de Wolfe está en la línea de lo que Sims denomina responsabilidad. El diálogo en periodismo narrativo ha levantado polémica, sobre todo, vinculada a las escenas explicadas en narrador omnisciente, por el nivel de responsabilidad que asume el autor y la adecuación ética de la situación. Para Sims se trata de responsabilidad con las personas de las que se habla. Según Wolfe (1973), se debe vivir el tiempo suficiente con ellas para presenciar las situaciones descritas. Para el autor, el diálogo es una técnica que se remonta a Balzac o Joyce. La prosa testimonial es otro afluente del periodismo narrativo (Angulo, 2013). Wolfe (1973) habla de figuras de finales del siglo XVII como Boswell. Reconoce A Journal of a Plague Year (1722) de Defoe, como el primer reportaje novelado.

Para Chillón (1999), la mayoría de edad del reportaje novelado llega a finales del siglo XIX, coincidiendo con el nacimiento de la prensa de masas. El periodismo como negocio y la necesidad de anuncios y lectores lleva a esta forma de narrar, que practican, durante los treinta, autores como Hersey. The New Yorker aparece como escuela (Herrscher, 2012) en la que firmas como Capote publicaron non-fiction novel. Wolfe o Talese forman la siguiente generación de autores de human interest stories (Chillón, 1999). También Kapuscinski o Guillermoprieto. Actualmente, Conover, Orlean o Bowden son periodistas narrativos. Hacen nuevo nuevo periodismo (Boynton, 2012) e incorporan elementos de las ciencias sociales.

\section{Del periodismo digital al periodismo narrativo digital}

El reportaje se ha estudiado exhaustivamente por su versatilidad. Larrondo (2009) señala cómo, a pesar de las posibilidades que el medio digital ofrece a los géneros periodísticos, éstos se ven obligados a acomodarse a ciertas particularidades.

Según Micó (2006), el estilo del periodismo digital se basa en: exactitud, claridad, concisión, densidad, precisión, sencillez, naturalidad, originalidad, brevedad, variedad, atracción, ritmo, color, sonoridad, detalle y propiedad. En 2001 se publicaron en Communication et langages, dos artículos que identificaban las características del ciberperiodismo, de Cotte (2001), Jeanne-Perrier (2001) y (Masip et al., 2010) que coinciden con las enunciadas por Micó (2006).

Este autor enumera también las propiedades del periodismo digital: datos actualizados, información universal, simultaneidad, versatilidad, proceso interactivo, multimedialidad e hipertextualidad. Deuze (2001), Pavlik (2001) o Rost (2006) han analizado el proceso interactivo y la participación. La hipertextualidad ha sido identificada como la característica más influyente en la construcción de discursos digitales (Larrondo, 2009).

Díaz Noci y Salaverría (2003) señalan que el texto digital es más profundo que largo, pero destacan que esta profundidad no debe afectar a la comprensión. Cabe diferenciar, según Larrondo (2009), un texto hipertextual de uno hipermedia. El primero contiene nódulos a otros textos; el segundo integra enlaces a elementos multimedia. La integración de estos elementos al periodismo narrativo es un tema de análisis académico, por las causas y consecuencias de esta hibridación de un género tradicional con otro innovador.

Albalad (2018) es uno de los autores que ha reflexionado sobre la versatilidad del reportaje de periodismo narrativo online en español en "Periodismo Slow o cómo se cuecen las historias en los fogones de Anfibia, Narratively y Frontera D", similar al presente. Albalad (2015), también ha analizado Longform.org basándose sobre todo en la 
entrevista como principal metodología. Con Rodríguez (Albalad; Rodríguez, 2012), estudia el llamado boom de revistas de periodismo narrativo y su rol digital.

En Iberoamérica, análisis semejantes son los de Puerta Molina (2016) en su estudio “La crónica latinoamericana actual: Io maravilloso real. Análisis del periodismo narrativo de Alberto Salcedo Ramos" o Salvatierra (2017) en "Notas para la investigación del periodismo literario en publicaciones digitales argentinas". En ambos casos, el tema de la investigación es parecido al de este artículo, aunque en el primer caso el análisis se centra en un solo autor, Alberto Salcedo, y en el segundo en un contexto, Argentina. Se detecta que los estudios más próximos lo son o bien porque analizan periodismo narrativo digital en otros contextos y a partir del análisis de otros medios o bien porque, a partir de una metodología similar a la presente, se habla del periodismo narrativo en un contexto concreto.

Entre las investigaciones similares en otras lenguas se detectan en Estados Unidos las aportaciones de Berning (2011) sobre las técnicas de los periodistas literarios en formato digital para crear verosimilitud. Bowden, en una entrevista con la autora, destaca cómo los reportajes de periodismo narrativo online son "verificablemente auténticos", porque añaden elementos complementarios que dan transparencia al relato. Berning (2011) añade que el nuevo periodismo era hipertextual antes de internet. Según la autora, el nivel de detalle al que llegan los escritores de periodismo narrativo les da hipertextualidad. El riesgo de que un enlace rompa la linealidad de la narración no es nuevo, una descripción detallada ya podía provocarlo. Berning confirma que el reportaje es de los géneros más adaptables al espacio digital.

También destacan en este campo las contribuciones de Dowling y Vogan (2015) sobre los efectos de la digitalización del reportaje longform a partir del referente Snowfall, de The New York Times (2012). Jacobson, Marino y Gutsche (2016) utilizan el análisis de contenido para evaluar el periodismo narrativo digital como género emergente. Le Masurier (2015), Belt y South (2016), Greenberg (2016), Neveu (2016) tratan el slow journalism.

En Finlandia, Lassila-Merisalo (2014), analiza en qué medida los recursos multimedia mejoran la narración entrevistando a periodistas de Narratively, Longreads, Informant, Zetland y Long Play en su estudio sobre el periodismo narrativo en el citado país. Van Krieken y Sanders (2016) utilizan el frame analysis y la entrevista para examinar el periodismo narrativo en los Países Bajos. En el mismo país, Drok y Hermans (2016) exploran el interés de los jóvenes por el slow journalism utilizando encuestas y el análisis cuantitativo. En Noruega, Larssen y Hornmoen (2013), estudian el contenido de dos novelas de no ficción para especificar las características del periodismo literario del país. En Canadá, Gillespie (2015) habla del periodismo narrativo a partir del análisis exhaustivo de una autora, Edna Staebler. En China, Guo (2014) examina el contenido de un reportaje de periodismo literario para localizar las particularidades del modelo en el país.

Además, autores como los siguientes repasan la evolución histórica del periodismo narrativo en distintos espacios geográficos. En Reino Unido Mckay (2011), Zdovc (2011) en Eslovenia, Soares (2011) en Portugal, Pereira Lima (2011) y Martínez (2017) en Brasil, y Hessell (2011) en Nueva Zelanda.

\section{Procedimientos Metodológicos}

La metodología de este estudio se basa en análisis de contenido, entrevista y observación. La elección la avalan técnicas utilizadas por investigaciones cercanas referentes. Las entrevistas en profundidad se han realizado a profesionales de medios de periodismo narrativo. Se ha hablado con responsables, redactores, colaboradores y expertos para estudiar el funcionamiento de estas publicaciones. Han sido entrevistas dirigidas.

La entrevista se ha utilizado en investigaciones cercanas por temática. Algunos ejemplos son Berning (2011) o Albalad y Rodríguez (2012). Además, la avalan los estudios de ciberperiodismo de Singer (1997) y Quandt, T. et al. (2003). Berning (2011) y Albalad y Rodríguez (2012) han analizado los portales de las revistas que configuran su muestra. El análisis de contenido lo han realizado autores como Paulussen (2004), Domingo y Heinonen (2008), y Jacobson, Marino y Gutsche (2016). 
La relación de entrevistas llevadas a cabo es: Robert Boynton, periodista en The New Yorker; Rubén Díaz, redactor de Jot Down; Ángel Fernández, director de Jot Down; Carles Foguet, director de comunicación de Jot Down; Leila Guerriero, editora de Gatopardo; Roberto Herrscher, experto en periodismo narrativo y colaborador de Gatopardo; Ricardo Jonás, subdirector de Jot Down; Emiliano Ruiz, redactor freelance de Gatopardo; Marcela Vargas, editora Web de Gatopardo; Julio Villanueva Chang, director de Etiqueta Negra. La selección de entrevistados se ha basado en el equilibrio entre medios y la variedad de cargos en cada uno de ellos. El objetivo ha sido contar con la máxima simetría de criterios a la vez que con una amplia variedad de puntos de vista sobre la temática. En este artículo se presenta una selección de las respuestas obtenidas con el consentimiento de todos los entrevistados. Se ha incorporado una síntesis de las más significativas y obviado las más particulares.

Se ha analizado una muestra de 30 artículos, 15 de Jot Down y 15 de Gatopardo. La selección se ha realizado de forma premeditada con el objetivo de que en la muestra fuera representativa y de que hubiera equilibro de artículos procedentes de distintas secciones y de distintos autores de cada uno de los medios. Se ha llegado a la cifra de 15 de cada uno teniendo en cuenta una media del número de secciones total. Todos son cercanos temporalmente. El Cuadro 1 muestra el equilibrio mencionado:

Cuadro 1. Clasificación de artículos analizados según medio, sección y autor.

\begin{tabular}{|c|c|c|c|}
\hline Artículo & Medio & Sección & Autor \\
\hline El reloj de Hildy & Jot Down & Opinión, Sociedad, Claroscuros, Columna & Enric González \\
\hline La luminosa magnesia de Manuel Alcántara & Jot Down & Columna, Opinión, ¿Qué tramáis morenos? & Jorge Bustos \\
\hline El misterio de Arnolfini, la joya robada & Jot Down & Arte y Letras & Dolores González Pastor \\
\hline $\begin{array}{l}\text { Las astutas y (perversas) tretas de los inquisidores } \\
\text { para lograr la confesión }\end{array}$ & Jot Down & Arte y Letras, Historia & Javier Bilbao \\
\hline Little Britain y el problema de la preposición & Jot Down & Cine y TV & Rubén Díaz Caviedes \\
\hline Sintonías que marcaron mi infancia & Jot Down & Cine y TV & Emilio Gorgot \\
\hline Vincenzo Nibali y la sombra del dopaje en el tour 2014 & Jot Down & Deportes & Guillermo Ortiz \\
\hline Brasil no se merecía esto & Jot Down & Deportes & Manuel de Lorenzo \\
\hline ¿Has visto mis gafas? & Jot Down & Ciencias, Ya lo veo & Pablo Artal \\
\hline Robocop y el desguace de la conciencia humana & Jot Down & Ciencias & José Valenzuela \\
\hline ¿Quién mató a Michael Hutchence? & Jot Down & Música & Jorge M. Molinero \\
\hline Julio Iglesias, el embajador universal & Jot Down & Música, Sociedad & Duncan Wheeler \\
\hline La enredadera de los muertos & Jot Down & Ocio y Vicio & Josep Lapidario \\
\hline $\begin{array}{l}\text { Ada Colau: "Lo mejor que puede hacer el PSOE } \\
\text { como estructura es desaparecer" }\end{array}$ & Jot Down & Entrevistas, Política y Economía & Cristian Campos \\
\hline $\begin{array}{l}\text { Peio Ruiz Cabestany: "Pasé el primero por el } \\
\text { Tourmalet y bajé llorando". }\end{array}$ & Jot Down & Deportes, Entrevistas & Ander Izaguirre \\
\hline La construcción de Golondrinas & Gatopardo & Reportajes & Emiliano Ruiz Parra \\
\hline Los señores de la costa & Gatopardo & Reportajes & Carlos Tello Díaz \\
\hline El hombre que fue Cantinflas & Gatopardo & Reportajes & Javier Molina \\
\hline The last ship & Gatopardo & Estilo de vida, Medios & Marcela Vargas \\
\hline Tipografía en escena & Gatopardo & Arquitectura y Diseño & Maru Aguzzi \\
\hline Todo menos "estándar" & Gatopardo & Estilos de vida, Viajes & Redacción \\
\hline El dragón blanco & Gatopardo & Estilo de vida, Tragos & Guillermo Sánchez Cervantes \\
\hline ¿Quién le teme a Avelina? & Gatopardo & Arte & $\begin{array}{l}\text { Guillermo Sánchez y Regina } \\
\text { Sienra }\end{array}$ \\
\hline Mercado gourmet & Gatopardo & Estilo de vida, Comida & Alejandra González Romo \\
\hline Pequeño pero poderoso & Gatopardo & Tecnología & Redacción \\
\hline Teatro para esperar y ser consolados & Gatopardo & Estilo de vida, Escena & Isabel Ibáñez de la Calle \\
\hline L.12.12 by Malafacha & Gatopardo & Estilo de vida, Moda & Alejandra González Romo \\
\hline Corazón de Florencia & Gatopardo & Estilo de vida, Compras & Redacción \\
\hline Cambio de paradigma & Gatopardo & Estilo de vida, Autos & Fabrizio Pozo \\
\hline Los números al poder & Gatopardo & Estilo de vida, Libros & Guillermo Sánchez Cervantes \\
\hline
\end{tabular}

Fuente: Elaborada por los autores (2018). 
Estas piezas han servido para recabar información sobre formato, uso de herramientas literarias, digitales, presencia en la red, además de para corroborar que la actividad corresponde con la planificación que anuncian en las entrevistas. Para ello, se ha utilizado una ficha que cuenta con cuatro partes: Identificación, Forma, Contenido y Audiencia. El primer apartado ubica la pieza dentro del medio, su sección, autor y titular. En el segundo se analizan los elementos, parte y estructura de cada pieza teniendo en cuenta la presencia y aspecto digital y en papel. Los campos que se detallan corresponden a los elementos a estudiar y son: subtítulos, complementos multimedia, imágenes, estrategias de posicionamiento y extensión, manifestada en número de scrolls y de páginas en papel. En cuanto al Contenido, en la ficha se profundiza en la narración. Por este motivo se identifica el tema y el tiempo verbal. Además, se revisa la presencia de las características principales que definen el periodismo narrativo: uso del diálogo, construcción escena por escena, descripciones realistas, uso de la primera/tercera persona, así como la presencia del periodista. Esta presencia se mide a partir de gradaciones: elevada (cuando la característica se repite reiteradamente en el texto), parcial (se repite más de cinco veces pero no está presente en la mayoría de párrafos) o baja (se detecta sólo en algún párrafo).

La parte de la ficha (Cuadro 2) que estudia la Audiencia registra el impacto de los artículos a partir de las reacciones provocadas en redes.

Cuadro 2. Ficha de análisis de los artículos.

\begin{tabular}{|c|c|}
\hline & ficación \\
\hline Número & Titular \\
\hline Autor & \\
\hline Sección & \\
\hline & rma \\
\hline Subtítulos & \\
\hline Elementos multimedia & \\
\hline Imagen & \\
\hline Elementos de posicionamiento & \\
\hline Scrolls & \\
\hline Páginas en papel & \\
\hline Caracteres & \\
\hline & enidos \\
\hline Tema & \\
\hline Persona & \\
\hline Tiempo verbal & \\
\hline Diálogo & \\
\hline Presencia del periodista & \\
\hline Punto de vista & \\
\hline Descripción & \\
\hline Uso de adjetivos & \\
\hline Construcción escena por escena & \\
\hline Otros detalles & \\
\hline & iencia \\
\hline Comentario & \\
\hline Redes en las que ha estado publicado & \\
\hline
\end{tabular}




\section{Resultados y Discusión}

La llegada del periodismo narrativo a internet ha supuesto una emergencia de medios nuevos en un momento muy próximo y por motivos que lo han facilitado:

1) Falta de cobertura de temas: estas revistas aparecen respondiendo a la insatisfacción con la oferta mediática. Lo confirman Foguet y Guerriero.

2) Facilidades tecnológicas: se han creado medios nuevos. Según Boynton, "the digital format is a great future for narrative journalism because the main problem for digital journalism has been how expensive it is to produce in the sense of reporting and research, so if you can cut the distribution costs it would help".

3) Internacionalización: el castellano une Latinoamérica y España y hace que el público potencial se amplíe.

4) Crisis económica: e inestabilidad política en ambos contextos, por las que la sociedad demanda medios independientes.

5) Cambio de paradigma comunicativo: los nuevos soportes suponen un reto.

\section{Contra la corriente digital}

El periodismo narrativo siempre ha sido polémico por romper normas (Wolfe, 1973). Muchas características de estilo del periodismo informativo se incumplen en estos reportajes; también las convenciones de la redacción online. Estos medios presentan formatos y rutinas que van contra lo óptimo en ciberperiodismo. Estos son los requerimientos del periodismo digital (Micó, 2006); se analiza en qué medida se incumplen:

1) Datos actualizados: el periodismo narrativo los utiliza, aunque su objetivo no es ser el primero en presentarlos (Herrscher, 2012).

2) Información universal: a pesar de que ambos medios tienen un público hispano, tratan temas universales. (Guerriero).

3) Simultaneidad: no es una prioridad. Los periodistas tienen tiempo para elaborar los reportajes (Herrscher, 2012) y lo agradecen porque lucen su obra (Rubén Díaz).

4) Interactividad: estos medios tienen contacto con el público y presencia en redes. La producción de contenidos no es interactiva. Según Foguet, el público interviene después, en la elaboración sólo se implican profesionales.

5) Multimedia: los medios de periodismo narrativo en formato digital no explotan al máximo los recursos multimedia (Berning, 2011). Para Herrscher, la protagonista es la narración.

6) Hipertexto: es de los más utilizados en los medios generalistas (Deuze, 2001). Los reportajes analizados de periodismo narrativo no lo usan frecuentemente. De los 30 artículos estudiados, 10 lo aplican.

7) Versatilidad: contribuye a la sostenibilidad del modelo, como explica Fernández. La aprovechan para promocionar otros productos vinculados a su cabecera.

Sobre las normas de estilo del periodismo digital (Micó, 2006), el periodismo narrativo cumple: exactitud, claridad, concisión, densidad, precisión, naturalidad, originalidad, variedad, atracción, ritmo, color, sonoridad, detalle y propiedad. Sin embargo, no es breve ni sencillo. Según Foguet y Guerriero, el criterio es la elaboración literaria. 
Cuadro 3. Resumen del análisis de los artículos según los criterios del periodismo narrativo [[Q1: Q1]].

1 del 2

\begin{tabular}{|c|c|c|c|c|c|c|c|c|}
\hline Artículo & Medio & Persona & Diálogo & $\begin{array}{l}\text { Presencia } \\
\text { periodista }\end{array}$ & Punto de vista & $\begin{array}{l}\text { Detalles } \\
\text { simbólicos }\end{array}$ & $\begin{array}{l}\text { Presencia } \\
\text { adjetivos }\end{array}$ & $\begin{array}{c}\text { Construcción } \\
\text { escena por } \\
\text { escena } \\
\end{array}$ \\
\hline El reloj de Hildy & Jot Down & $\begin{array}{l}\text { Primera singular y } \\
\text { plural }\end{array}$ & Parcial & Elevada & $\begin{array}{l}\text { Narrador } \\
\text { protagonista }\end{array}$ & Parcial & Parcial & Parcial \\
\hline $\begin{array}{l}\text { La luminosa } \\
\text { magnesia de Manuel } \\
\text { Alcántara }\end{array}$ & Jot Down & $\begin{array}{l}\text { Primera singular y } \\
\text { plural }\end{array}$ & No & Elevada & $\begin{array}{l}\text { Narrador } \\
\text { protagonista }\end{array}$ & Elevada & Elevada & Elevada \\
\hline $\begin{array}{l}\text { El misterio de } \\
\text { Arnolfini, la joya } \\
\text { robada }\end{array}$ & Jot Down & $\begin{array}{l}\text { Primera plural y } \\
\text { Tercera singular }\end{array}$ & Parcial & Baja & Narrador externo & Elevada & Elevada & Elevada \\
\hline $\begin{array}{l}\text { Las astutas y } \\
\text { (perversas) tretas de } \\
\text { los inquisidores para } \\
\text { lograr la confesión }\end{array}$ & Jot Down & $\begin{array}{l}\text { Tercera singular y } \\
\text { plural y Segunda } \\
\text { plural }\end{array}$ & Parcial & Parcial & Narrador externo & Elevada & Parcial & Elevada \\
\hline $\begin{array}{l}\text { Little Britain y el } \\
\text { problema de la } \\
\text { preposición }\end{array}$ & Jot Down & $\begin{array}{l}\text { Tercera singular y } \\
\text { plural y Segunda } \\
\text { plural }\end{array}$ & Parcial & Parcial & Narrador externo & Parcial & Parcial & Parcial \\
\hline $\begin{array}{l}\text { Sintonías que } \\
\text { marcaron mi infancia }\end{array}$ & Jot Down & Primera singular & Parcial & Elevada & $\begin{array}{l}\text { Narrador } \\
\text { protagonista }\end{array}$ & Parcial & Parcial & Elevada \\
\hline $\begin{array}{l}\text { Vincenzo Nibali y la } \\
\text { sombra del dopaje } \\
\text { en el tour } 2014\end{array}$ & Jot Down & Tercera singular & Parcial & Parcial & Narrador externo & Parcial & Parcial & Parcial \\
\hline $\begin{array}{l}\text { Brasil no se merecía } \\
\text { esto }\end{array}$ & Jot Down & Primera singular & Parcial & Elevada & $\begin{array}{l}\text { Narrador } \\
\text { omnisciente }\end{array}$ & Elevada & Elevada & Elevada \\
\hline ¿Has visto mis gafas? & Jot Down & Primera singular & Parcial & Elevada & $\begin{array}{l}\text { Narrador } \\
\text { protagonista }\end{array}$ & Parcial & Parcial & Parcial \\
\hline $\begin{array}{l}\text { Robocop y el } \\
\text { desguace de la } \\
\text { conciencia humana }\end{array}$ & Jot Down & $\begin{array}{l}\text { Primera singular } \\
\text { y plural y Tercera } \\
\text { singular }\end{array}$ & Sí & Baja & Narrador externo & Elevada & Parcial & Elevada \\
\hline $\begin{array}{l}\text { ¿Quién mató a } \\
\text { Michael Hutchence? }\end{array}$ & Jot Down & $\begin{array}{l}\text { Primera singular y } \\
\text { Tercera singular }\end{array}$ & No & Parcial & $\begin{array}{l}\text { Narrador } \\
\text { omnisciente y } \\
\text { externo }\end{array}$ & Elevada & Elevada & Parcial \\
\hline $\begin{array}{l}\text { Julio Iglesias, el } \\
\text { embajador universal }\end{array}$ & Jot Down & Primera singular & No & Elevada & $\begin{array}{l}\text { Narrador } \\
\text { protagonista }\end{array}$ & Elevada & Elevada & Elevada \\
\hline $\begin{array}{l}\text { La enredadera de los } \\
\text { muertos }\end{array}$ & Jot Down & $\begin{array}{l}\text { Primera singular y } \\
\text { Tercera singular y } \\
\text { plural }\end{array}$ & No & Elevada & $\begin{array}{l}\text { Narrador } \\
\text { protagonista }\end{array}$ & Elevada & Elevada & Elevada \\
\hline $\begin{array}{l}\text { Ada Colau: "Lo } \\
\text { mejor que puede } \\
\text { hacer el PSOE } \\
\text { como estructura es } \\
\text { desaparecer }\end{array}$ & Jot Down & $\begin{array}{l}\text { Segunda singular y } \\
\text { Tercera singular }\end{array}$ & Sí & Parcial & $\begin{array}{l}\text { Narrador } \\
\text { protagonista }\end{array}$ & Elevada & Elevada & No \\
\hline $\begin{array}{l}\text { Peio Ruiz Cabestany: } \\
\text { "Pasé el primero } \\
\text { por el } \\
\text { Tourmalet y bajé } \\
\text { "lorando" }\end{array}$ & Jot Down & $\begin{array}{l}\text { Segunda singular y } \\
\text { Tercera singular }\end{array}$ & Sí & Parcial & $\begin{array}{l}\text { Narrador } \\
\text { protagonista }\end{array}$ & Elevada & Baja & Parcial \\
\hline $\begin{array}{l}\text { La construcción de } \\
\text { Golondrinas }\end{array}$ & Gatopardo & $\begin{array}{l}\text { Primera singular y } \\
\text { tercera singular }\end{array}$ & Parcial & Elevada & $\begin{array}{l}\text { Narrador } \\
\text { protagonista y } \\
\text { omnisciente }\end{array}$ & Elevada & Elevada & Elevada \\
\hline $\begin{array}{l}\text { Los señores de la } \\
\text { costa }\end{array}$ & Gatopardo & Tercera plural & Parcial & Baja & $\begin{array}{l}\text { Narrador externo } \\
\text { y omnisciente }\end{array}$ & Elevada & Elevada & Elevada \\
\hline
\end{tabular}

Fuente: Elaborada por los autores (2018). 
Cuadro 3. Resumen del análisis de los artículos según los criterios del periodismo narrativo [[Q1: Q1]]

\begin{tabular}{|c|c|c|c|c|c|c|c|c|}
\hline Artículo & Medio & Persona & Diálogo & $\begin{array}{l}\text { Presencia } \\
\text { periodista }\end{array}$ & Punto de vista & $\begin{array}{l}\text { Detalles } \\
\text { simbólicos }\end{array}$ & $\begin{array}{l}\text { Presencia } \\
\text { adjetivos }\end{array}$ & $\begin{array}{c}\text { Construcción } \\
\text { escena por } \\
\text { escena }\end{array}$ \\
\hline $\begin{array}{l}\text { El hombre que fue } \\
\text { Cantinflas }\end{array}$ & Gatopardo & $\begin{array}{l}\text { Primera singular y } \\
\text { Tercera singular }\end{array}$ & Parcial & Elevada & $\begin{array}{l}\text { Narrador } \\
\text { omnisciente }\end{array}$ & Elevada & Elevada & Elevada \\
\hline The last ship & Gatopardo & Tercera singular & Sí & Parcial & Narrador externo & Parcial & Parcial & Parcial \\
\hline Tipografía en escena & Gatopardo & Tercera singular & No & Baja & Narrador externo & Elevada & Elevada & Elevada \\
\hline $\begin{array}{l}\text { Todo menos } \\
\text { "estándar" }\end{array}$ & Gatopardo & Tercera singular & No & Baja & Narrador externo & Elevada & Elevada & Baja \\
\hline El dragón blanco & Gatopardo & Tercera singular & Parcial & Parcial & Narrador externo & Elevada & Elevada & Parcial \\
\hline $\begin{array}{l}\text { ¿Quién le teme a } \\
\text { Avelina? }\end{array}$ & Gatopardo & Tercera singular & Parcial & Baja & Narrador externo & Elevada & Elevada & Parcial \\
\hline Mercado gourmet & Gatopardo & $\begin{array}{l}\text { Tercera singular y } \\
\text { plural }\end{array}$ & No & Parcial & Narrador externo & Elevada & Elevada & Elevada \\
\hline $\begin{array}{l}\text { Pequeño pero } \\
\text { poderoso }\end{array}$ & Gatopardo & $\begin{array}{l}\text { Tercera singular y } \\
\text { primera plural }\end{array}$ & No & Baja & Narrador externo & Elevada & Parcial & No \\
\hline $\begin{array}{l}\text { Teatro para esperary } \\
\text { ser consolados }\end{array}$ & Gatopardo & Primera singular & No & Elevada & $\begin{array}{l}\text { Narrador } \\
\text { protagonista }\end{array}$ & Elevada & Parcial & Parcial \\
\hline L.12.12 by Malafacha & Gatopardo & Tercera plural & No & No & Narrador externo & Parcial & Parcial & No \\
\hline Corazón de Florencia & Gatopardo & $\begin{array}{l}\text { Tercera singular y } \\
\text { plural }\end{array}$ & No & Baja & Narrador externo & Parcial & Parcial & No \\
\hline $\begin{array}{l}\text { Cambio de } \\
\text { paradigma }\end{array}$ & Gatopardo & Tercera singular & No & No & Narrador externo & Elevada & Parcial & No \\
\hline Los números al poder & Gatopardo & Tercera singular & No & Baja & $\begin{array}{l}\text { Narrador } \\
\text { omnisciente }\end{array}$ & Parcial & Parcial & Parcial \\
\hline
\end{tabular}

Fuente: Elaborada por los autores (2018).

\section{De fast journalism a slow journalism}

El periodismo narrativo incumple también la norma de inmediatez de las publicaciones digitales. La base del periodismo narrativo digital es la filosofía slow, que defiende una producción y consumo tecnológicos reflexivos y humanos (Barranquero-Carretero, 2013). Este ritmo se aplica a la producción de contenidos y a la actualización digital. Boynton asegura: "We are trained to instant production. My definition of narrative journalism is something that takes a long time". Los editores de Jot Down y Gatopardo son conscientes de que su papel no es publicar primicias.

Se da un enfoque de reflexión para analizar las consecuencias de los hechos. Esta postura respecto a la actualidad se muestra también en la ausencia de la fecha en los artículos. Guerriero asegura que estos medios pueden tomarse estas licencias porque el lector es consciente de esta temporalidad. Existe un pacto con el público; los lectores obtienen calidad y los periodistas, unas condiciones de trabajo sin prisas.

Esta voluntad de frenar el fast journalism digital se refleja también en el rol de las redes sociales en estos medios. Son un espacio donde difundir y medir el éxito de los contenidos se consideran parte integral del medio y una vía de contacto con los lectores. Los seguidores comparten y comentan los contenidos, pero no intervienen en la producción.

\section{Fidelidad al Nuevo Periodismo}

A pesar de romper moldes del periodismo informativo y del ciberperiodismo, el periodismo narrativo digital es fiel al periodismo narrativo tradicional. Las características de este tipo de periodismo son inherentes al reportaje, el género más maleable ante este tipo de narración (Chillón, 1999). Vivaldi (1999) lo define como: "Una información de altos vuelos, con más libertad expositiva [...]. El reportaje es un relato informativo, una narración más o menos 
noticiosa en donde la visión personal del periodista, su modo de enfocar el asunto, influyen en la concepción del trabajo". Sin citar el periodismo narrativo, el autor lo define al explicar el reportaje: libertad expositiva, narración noticiosa y visión personal del periodista. Los artículos analizados en esta investigación son una prueba de lo enunciado. En ellos, se encuentran los elementos del nuevo periodismo según Wolfe (1973):

1) Construcción escena por escena: en 25 de los 30 artículos analizados. En 13 a un nivel elevado, en 11 parcial y en 1 bajo. Esta característica es la más vinculada al hipertexto, pues rompe una narración lineal (Berning, 2011).

2) Diálogo realista: en 16 de 30 artículos. Esta característica es representativa del periodismo narrativo y la que ha creado más polémica.

3) Detalles simbólicos: las descripciones detalladas presentan debate ético pero permiten introducir al lector en la narración. Se encuentran en los 30 artículos. En 21 aparecen en un grado elevado y en 9 parcial.

4) Tercera persona: mayoritaria junto con la primera en todos los artículos; en singular y en plural como en omnisciente. Guerriero y Herrscher defienden esta técnica como el modo de ofrecer al lector un punto de vista nuevo.

\section{Papelización de la Web}

Más allá de las rutinas que el periodismo narrativo rompe en el medio digital, el análisis permite comprobar el desaprovechamiento que hace de los recursos digitales. Albalad y Rodríguez (2012) lo aprecian en su análisis de Frontera D. Deuze (2001), Canavilhas (2007) y (Masip et al. (2010), señalan que algunos elementos multimedia complementan el texto por yuxtaposición.

La papelización de la Web (Rodríguez; Albalad, 2013) es un fenómeno que resume esta fidelidad a la tradición por parte del periodismo narrativo. Se da en Jot Down y en Gatopardo, a pesar de que la primera naciera en el medio digital y se editara después en papel y la segunda hiciera lo contrario. Para Foguet esta papelización es intencionada. Las principales características de esta papelización son:

1) Protagonismo del texto: por el uso de la narración como protagonista y una ausencia de complementos multimedia. Éstos no suelen estar integrados en los temas. Así, en Gatopardo existe la sección "Multimedia" en la que se sitúan los artículos que se trabajan con más complementos digitales.

2) Portada papel: la maquetación de estas revistas digitales está muy vinculada a la versión papel. El ejemplo es la portada digital de Gatopardo, con el mismo formato que en papel.

3) Estructura numerada: a pesar de no detallar la fecha de publicación, los artículos de estas revistas en formato digital dejan entrever a qué número del papel pertenece cada publicación. En Jot Down, los situados en la sección "Lo más nuevo", son los publicados en el número en papel más reciente.

4) Referencias al papel: con secciones como la hemeroteca o elementos a la venta como complementos al papel.

5) Reconocimiento del autor: a diferencia de los medios informativos en formato digital, en estos medios el autor tiene relevancia.

En los medios de periodismo narrativo digital se refleja que el papel no queda atrás. En ambas publicaciones, además de la papelización de la Web, la edición en papel adopta un rol superior. Con un formato elaborado, se aporta valor al contenido. Este valor se traduce en su precio, cercano al de un libro. Los dos formatos ofrecen valor añadido al público: el papel, la elaboración y la Web, la gratuidad.

\section{Periodismo narrativo digital: la empresa}

Los medios de periodismo narrativo digital se integran en la empresa en modelos de negocio híbridos. Jot Down o Gatopardo necesitan formar parte de una empresa que cuente con más vías de ingreso. Gatopardo está dentro de Travesías Media, una empresa que tiene más publicaciones y que ofrece servicios de comunicación. 
Guerriero señala que es complejo que una revista así sea viable por ella misma. Jot Down, es un producto de la empresa Wabi Sabi Investments; cuenta con la editorial Jot Down Books, por la cual distribuyen productos propios y de otras editoriales. Realizan también servicios de gestión cultural bajo la marca Tanyible y venden contenidos a revistas internacionales. Desde 2015, tienen un acuerdo con El País, por el cual mensualmente se publica Jot Down Smart, una versión reducida con contenidos disponibles en digital pero editados para papel.

Este modelo se traduce en una situación laboral específica para los periodistas. Por un lado, se abren las puertas a nuevos profesionales gracias al medio digital; este hecho facilita el descubrimiento de firmas nuevas (Guerriero). Para el profesional trabajar en estas plataformas supone un reconocimiento. Las condiciones, según Emiliano Ruiz, son flexibles. Trabajo a fuego lento, sin presiones y amplia difusión. Sin embargo, las plantillas de los medios analizados están formadas por periodistas freelance. La estructura logística central la configuran un número reducido de profesionales contratados. En Gatopardo, éstos cuentan con una localización física. En Jot Down la redacción no existe. Para Julio Villanueva, de Etiqueta Negra se puede vivir del periodismo narrativo, aunque sólo algunos son capaces de hacerlo porque "es una competencia muy exigente y los más conmovedores, inteligentes, comprometidos y arriesgados son una minoría de la minoría" (Cuadro 4).

Cuadro 4. Comparación de las opiniones de los entrevistados.

\begin{tabular}{|c|c|c|c|c|c|c|c|}
\hline Autores & $\begin{array}{l}\text { Periodismo } \\
\text { narrativo }\end{array}$ & $\begin{array}{c}\text { Periodista } \\
\text { narrativo }\end{array}$ & Papel vs. Online & $\begin{array}{l}\text { Elementos } \\
\text { multimedia }\end{array}$ & $\begin{array}{c}\text { Elemento } \\
\text { característico } \\
\text { de su medio }\end{array}$ & $\begin{array}{l}\text { Interacción } \\
\text { lectores }\end{array}$ & Viabilidad \\
\hline Robert Boynton & $\begin{array}{l}\text { La forma } \\
\text { siempre es lo } \\
\text { primero. }\end{array}$ & $\begin{array}{l}\text { Curiosidad y } \\
\text { resistencia. }\end{array}$ & $\begin{array}{l}\text { La plataforma } \\
\text { online ayuda } \\
\text { a difundir los } \\
\text { contenidos y } \\
\text { elimina costes. }\end{array}$ & $\begin{array}{l}\text { Pueden ser una } \\
\text { distracción. }\end{array}$ & & & $\begin{array}{l}\text { En este } \\
\text { momento la } \\
\text { gran mayoría } \\
\text { de periodistas } \\
\text { narrativos } \\
\text { tienen que } \\
\text { trabajar en algo } \\
\text { más. }\end{array}$ \\
\hline $\begin{array}{l}\text { Rubén Díaz } \\
\text { Caviedes }\end{array}$ & $\begin{array}{l}\text { Elaboración } \\
\text { literaria, contar } \\
\text { las cosas de } \\
\text { manera distinta. }\end{array}$ & & $\begin{array}{l}\text { Los contenidos } \\
\text { Web los } \\
\text { contenidos son } \\
\text { más accesibles. }\end{array}$ & $\begin{array}{l}\text { Bajo, la } \\
\text { importancia } \\
\text { está en el texto. }\end{array}$ & $\begin{array}{l}\text { Una de las } \\
\text { cosas que más } \\
\text { le gustan en } \\
\text { periodismo es } \\
\text { poder tardar. }\end{array}$ & $\begin{array}{l}\text { Tiene en cuenta } \\
\text { las opiniones } \\
\text { del público } \\
\text { dependiendo } \\
\text { de quién } \\
\text { vengan. }\end{array}$ & $\begin{array}{l}\text { Si no cobrara } \\
\text { no le podría } \\
\text { dedicar } \\
\text { el tiempo } \\
\text { necesario. }\end{array}$ \\
\hline $\begin{array}{l}\text { Ángel Luis } \\
\text { Fernández }\end{array}$ & & & & & & & $\begin{array}{l}\text { Ajustan en } \\
\text { gastos a lo } \\
\text { que tienen de } \\
\text { ingresos. }\end{array}$ \\
\hline Carles Foguet & $\begin{array}{l}\text { Rechazo a la } \\
\text { superficialidad } \\
\text { y cariño por } \\
\text { la calma. Se } \\
\text { publica todo } \\
\text { lo que se } \\
\text { explique con } \\
\text { vocación de } \\
\text { calidad literaria, } \\
\text { con pasión de } \\
\text { quien escribe. }\end{array}$ & $\begin{array}{l}\text { Amplitud } \\
\text { de miras, } \\
\text { profundidad de } \\
\text { conocimiento } \\
\text { y pasión por lo } \\
\text { que se escribe. }\end{array}$ & $\begin{array}{l}\text { Los contenidos } \\
\text { digitales no son } \\
\text { los mismos que } \\
\text { los de papel. La } \\
\text { actualidad no } \\
\text { es un valor. }\end{array}$ & $\begin{array}{l}\text { Conscientes de } \\
\text { no explotar las } \\
\text { posibilidades } \\
\text { del medio, son } \\
\text { la translación } \\
\text { del papel a la } \\
\text { red. }\end{array}$ & $\begin{array}{l}\text { La revista es } \\
\text { una macedonia, } \\
\text { un mosaico de } \\
\text { contenido. }\end{array}$ & $\begin{array}{l}\text { A través de las } \\
\text { redes sociales. } \\
\text { Atentos a } \\
\text { la suma de } \\
\text { interacciones } \\
\text { que llegan } \\
\text { por distintos } \\
\text { canales. }\end{array}$ & $\begin{array}{l}\text { Si Jot Down no } \\
\text { fuera viable no } \\
\text { existiría. }\end{array}$ \\
\hline
\end{tabular}

Fuente: Elaborada por los autores (2018). 
Cuadro 4. Comparación de las opiniones de los entrevistados.

\begin{tabular}{|c|c|c|c|c|c|c|c|}
\hline Autores & $\begin{array}{l}\text { Periodismo } \\
\text { narrativo }\end{array}$ & $\begin{array}{c}\text { Periodista } \\
\text { narrativo }\end{array}$ & Papel vs. Online & $\begin{array}{l}\text { Elementos } \\
\text { multimedia }\end{array}$ & $\begin{array}{c}\text { Elemento } \\
\text { característico } \\
\text { de su medio }\end{array}$ & $\begin{array}{l}\text { Interacción } \\
\text { lectores }\end{array}$ & Viabilidad \\
\hline Leila Guerriero & $\begin{array}{l}\text { Singularidad } \\
\text { universal. }\end{array}$ & $\begin{array}{l}\text { Capacidad } \\
\text { de reporteo, } \\
\text { mirada propia } \\
\text { y escritura } \\
\text { excelente. }\end{array}$ & $\begin{array}{l}\text { Mensual en } \\
\text { papel, temas } \\
\text { atemporales } \\
\text { conviven con } \\
\text { temas más } \\
\text { actuales en } \\
\text { la versión } \\
\text { digital. Los } \\
\text { temas largos } \\
\text { en ambos } \\
\text { soportes. }\end{array}$ & & $\begin{array}{l}\text { Sofisticación } \\
\text { de contar las } \\
\text { historias bien } \\
\text { contadas. }\end{array}$ & $\begin{array}{l}\text { Sólo a través de } \\
\text { email y redes } \\
\text { sociales, no en } \\
\text { los procesos de } \\
\text { producción. }\end{array}$ & $\begin{array}{l}\text { Es difícil que la } \\
\text { revista funcione } \\
\text { sola, se sostiene } \\
\text { dentro de un } \\
\text { esquema de } \\
\text { negocio. }\end{array}$ \\
\hline $\begin{array}{l}\text { Roberto } \\
\text { Herrscher }\end{array}$ & $\begin{array}{l}\text { Estas revistas } \\
\text { cumplen } \\
\text { dos normas } \\
\text { del buen } \\
\text { periodismo: } \\
\text { novedad y } \\
\text { profundidad. }\end{array}$ & $\begin{array}{l}\text { Es un desafío } \\
\text { que lleva a } \\
\text { investigar } \\
\text { mucho, a auto } \\
\text { editar. }\end{array}$ & $\begin{array}{l}\text { Uno busca una } \\
\text { historia bien } \\
\text { contada en } \\
\text { palabras. }\end{array}$ & $\begin{array}{l}\text { Considera el } \\
\text { multimedia irse } \\
\text { por las ramas. }\end{array}$ & $\begin{array}{l}\text { Tiene ambición } \\
\text { de calidad, } \\
\text { vocación de } \\
\text { permanencia. }\end{array}$ & & \\
\hline Ricardo Jonás & $\begin{array}{l}\text { Que el tema } \\
\text { sea interesante } \\
\text { y esté bien } \\
\text { escrito. }\end{array}$ & & $\begin{array}{l}\text { En Web la ve } \\
\text { más gente, } \\
\text { en papel sólo } \\
\text { las personas } \\
\text { que compran } \\
\text { la revista. La } \\
\text { edición Web es } \\
\text { más rápida que } \\
\text { en papel. }\end{array}$ & & $\begin{array}{l}\text { Mucha } \\
\text { importancia a la } \\
\text { forma y a que el } \\
\text { contenido esté } \\
\text { bien escrito. }\end{array}$ & $\begin{array}{l}\text { En las redes } \\
\text { sociales hay } \\
\text { muchos } \\
\text { lectores } \\
\text { habituales } \\
\text { que de vez } \\
\text { en cuando } \\
\text { comentan un } \\
\text { artículo. }\end{array}$ & \\
\hline $\begin{array}{l}\text { Emiliano Ruiz } \\
\text { Parra }\end{array}$ & & $\begin{array}{l}\text { Es un espacio } \\
\text { donde } \\
\text { puede tratar } \\
\text { prácticamente } \\
\text { cualquier } \\
\text { tema con total } \\
\text { libertad. }\end{array}$ & $\begin{array}{l}\text { Para él, placer } \\
\text { es publicar en } \\
\text { revista impresa. }\end{array}$ & $\begin{array}{l}\text { La apuesta es } \\
\text { la fortaleza } \\
\text { del texto y la } \\
\text { belleza de las } \\
\text { fotografías. }\end{array}$ & $\begin{array}{l}\text { Gatopardo es } \\
\text { un espacio de } \\
\text { lucimiento. }\end{array}$ & $\begin{array}{l}\text { A través de } \\
\text { Twitter y de los } \\
\text { comentarios } \\
\text { que dejan los } \\
\text { lectores. }\end{array}$ & $\begin{array}{l}\text { Uno tiene } \\
\text { que tener dos } \\
\text { empleos, el } \\
\text { que le permita } \\
\text { comer y el } \\
\text { que le permita } \\
\text { escribir. }\end{array}$ \\
\hline Marcela Vargas & $\begin{array}{l}\text { En la versión } \\
\text { digital de } \\
\text { Gatopardo son } \\
\text { los mismos que } \\
\text { en la versión en } \\
\text { papel. }\end{array}$ & & $\begin{array}{l}\text { La Web tiene } \\
\text { contenido } \\
\text { exclusivo, que } \\
\text { por formato o } \\
\text { temporalidad } \\
\text { no cabe en } \\
\text { la versión } \\
\text { impresa. Las } \\
\text { secciones } \\
\text { culturales y de } \\
\text { estilo de vida se } \\
\text { actualizan cada } \\
\text { día. El reportaje } \\
\text { o crónica de } \\
\text { portada se } \\
\text { actualiza la } \\
\text { penúltima } \\
\text { semana de } \\
\text { mes. }\end{array}$ & & & $\begin{array}{l}\text { Los } \\
\text { comentarios } \\
\text { de los lectores } \\
\text { permiten tomar } \\
\text { la temperatura } \\
\text { de los temas. }\end{array}$ & \\
\hline
\end{tabular}


Cuadro 4. Comparación de las opiniones de los entrevistados.

\begin{tabular}{|c|c|c|c|c|c|c|c|}
\hline Autores & $\begin{array}{c}\text { Periodismo } \\
\text { narrativo }\end{array}$ & $\begin{array}{c}\text { Periodista } \\
\text { narrativo }\end{array}$ & Papel vs. Online & $\begin{array}{l}\text { Elementos } \\
\text { multimedia }\end{array}$ & $\begin{array}{c}\text { Elemento } \\
\text { característico } \\
\text { de su medio }\end{array}$ & $\begin{array}{l}\text { Interacción } \\
\text { lectores }\end{array}$ & Viabilidad \\
\hline $\begin{array}{l}\text { Julio Villanueva } \\
\text { Chang }\end{array}$ & $\begin{array}{l}\text { Trabajo de } \\
\text { investigación, } \\
\text { intelectual y de } \\
\text { interpretación. }\end{array}$ & $\begin{array}{l}\text { Curiosidad, } \\
\text { ganas de } \\
\text { conocer el } \\
\text { mundo y a la } \\
\text { gente. }\end{array}$ & $\begin{array}{l}\text { No tienen } \\
\text { versión digital } \\
\text { porque la } \\
\text { consideran una } \\
\text { distracción. }\end{array}$ & $\begin{array}{l}\text { No tienen } \\
\text { versión digital. }\end{array}$ & $\begin{array}{l}\text { Publican } \\
\text { grandes temas } \\
\text { que adoptan } \\
\text { mil caras. }\end{array}$ & No influye. & $\begin{array}{l}\text { Sólo puede vivir } \\
\text { del periodismo } \\
\text { narrativo una } \\
\text { minoría. }\end{array}$ \\
\hline
\end{tabular}

Fuente: Elaborada por los autores (2018).

\section{Conclusión}

El periodismo narrativo digital es efectivo y permite tener lectores. Su emergencia ha abierto la puerta a nuevos medios en el mundo iberoamericano y en el espacio mediático anglosajón. Éstos han aparecido para ocupar nichos temáticos y gracias a las facilidades de las plataformas digitales. Con el paso a formato digital de medios tradicionales, estos nuevos medios aparecen como un concepto distinto ante el lector. Se basan en la voluntad de calidad literaria y en soportes elaborados que contribuyen a crear una marca consolidada que quiere llegar mucho más allá que la cabecera de un medio.

Jot Down y Gatopardo son muestras de esta situación. Ambos han creado una simbiosis entre sus versiones digital y papel. En el espacio digital, estas revistas ponen en práctica el nuevo periodismo. Destacan a su autor como el artista que está detrás de una obra que tiene la voluntad de ser literaria.

Las rutinas que siguen este tipo de medios se pueden englobar en el movimiento slow. Sus vínculos son más fuertes con las humanidades que con la tecnología, aunque los periodistas narrativos son conscientes de que no pueden desarrollarse sin ella. El reportaje digital se presenta como un espacio en el que caben elementos digitales combinados con un periodismo narrativo austero en el uso de éstos e infiel a las normas del ciberperiodismo; a la vez, sigue las características del periodismo literario tradicional: construcción escena por escena, diálogo realista, detalles simbólicos, narrador en primera o tercera persona. El análisis de 30 artículos y las entrevistas realizadas lo demuestran.

Se detecta una papelización de la Web que se muestra en las versiones digitales de los medios analizados. Protagonismo del texto, portada papel, estructura numerada, referencias al papel y reconocimiento del autor son los elementos de esta papelización. Por ahora, los medios de periodismo narrativo digital no son viables individualmente; se desarrollan bajo estructuras empresariales más amplias que comercializan productos relacionados, como libros. Son empresas que ofrecen condiciones laborales flexibles pero poco estables; gran parte de los autores de los reportajes son freelance que trabajan desde cualquier parte del mundo.

Así, se identifica una evolución del nuevo periodismo de Wolfe y del nuevo nuevo periodismo de Boynton, que se presenta con un equilibrio entre tradición e innovación; tiene en cuenta humanidades y ciencias sociales en un entorno digital que incorpora las características del papel. Al mismo tiempo, tiene la voluntad de incrementar el prestigio del papel y del periodista, a quien reconoce como autor. Así, el nuevo nuevo nuevo periodismo ha realizado su revolución y los primeros resultados demuestran que viene para quedarse.

\section{Colaboradores}

Todos los autores han contribuido en todas las etapas de la investigación, explotación y análisis de datos y redacción final. 


\section{Referencias}

Albalad, J.M. Slow journalism para una nueva audiencia digital: el caso de Longform.org (2010-2015). Revista de Comunicación, n.14, p.7-25, 2015.

Albalad, J.M. Periodismo slow. Madrid: Fragua, 2018.

Albalad, J.M.; Rodríguez, J.M. New perspectives on Spanish language narrative journalism. Textual and Visual Media, v.5, n.5, p.115-138, 2012.

Angulo, M. Crónica y Mirada: aproximaciones al periodismo narrativo. Madrid: Libros del K.O., 2013.

Bak, J.S.; Reynolds, B. Literary Journalism across the globe. Boston: University of Massachusetts Press, 2011.

Barranquero-Carretero, A. Slow Media: comunicación, cambio social y sostenibilidad en la era del torrente mediático. Palabra Clave, v.16, n.2, p.419-448, 2013.

Belt, D.; South, J. Slow Journalism and the out of the Eden walk. Digital Journalism, v.4, n.4, p.547-562, 2016. http://dx.doi.org/1 $0.1080 / 21670811.2015 .1111768$

Berning, N. Narrative Journalism in the Age of the Internet new ways to create authencity in online literary reportages. Textpraxis, n.3, p.1-13, 2011. http://dx.doi.org/10.17879/57289624008

Boynton, R. El nuevo Nuevo Periodismo. Barcelona: Universitat de Barcelona Publicacions i Edicions, 2012.

Canavilhas, J. Webnoticia: propuesta de modelo periodístico para la WWW. Covilhã: LabCom Books, 2007.

Capote, T. A sangre fría. Madrid: Anagrama, 1965.

Chillón, A. Literatura y Periodismo: una tradición de relaciones promiscuas. Barcelona: Universitat Autònoma de Barcelona, 1999.

Cotte, D. De la Une à l'écran, avatars du texte journalistique. Communication et Langages, n.129, p.64-78, 2001.

Deuze, M. Online journalism: Modelling the first generation of news media in the World Wide Web. First Monday, v.6, n.10, 2001.

Díaz Noci, J.; Salaverría, R. Manual de redacción ciberperiodística. Barcelona: Ariel Comunicación, 2003.

Domingo, D.; Heinonen, A. Weblogs and Journalism. Nordicom Review, v.29, n.1, p.3-15, 2008.

Dowling, D.; Vogan, T. Can we "snowfall" this? Journal Digital Journalism, v.3, n.2, p.209-224, 2015. http://dx.doi.org/10.108 0/21670811.2014.930250

Drok, N.; Hermans, L. Is there a future for slow journalism? Journalism Practice, v.10, n.4, p.539-554, 2016.

Franklin, J. Writing for a story. New York: Penguin Books/Plum, 1986.

Franklin, J. When to go long. American Journalism Review, v.18, n.10, p.36-40, 1996.

Gillespie, B. The works of Edna Staebler: Using Literary Journalism to celebrate the lives of ordinary Canadians. Literary Journalism Studies, v.7, n.1, p.59-75, 2015.

Greenberg, S. Editing, fast and slow. Journalism Practice, v.10, n.4, p.555-567, 2016. http://dx.doi.org/10.1080/17512786.20 15.1114898
Guo, Z. Advancing a typology of media content: Analysis of a Literary Journalism story. Journal of Mass Communication \& Journalism, v.5, n.1, p.1-8, 2014. http://dx.doi.org/10.4172/21657912.1000237

Gutiérrez Palacio, J. De Azorín a Umbral: un siglo de periodismo literario español. La Coruña: Netbiblo, 2009.

Hartsock, J. A History of American Literary Journalism: The emergence of a modern narrative form. Boston: University of Massachusetts Press, 2001.

Herrscher, R. Periodismo narrativo: cómo contar la realidad con las armas de la literatura. Barcelona: Universitat Autònoma de Barcelona, 2012.

Hessell, N. Riding the rails with Robin Hyde: Literary Journalism in the 1930s New Zealand. In: Bak, J.S.; Reynolds, B. (Ed.). Literary Journalism across the globe. Amherst and Boston: University of Massachusetts Press, 2011.

International Association for Literary Journalism Studies. Mission Statement. 2006. Available from: <http://ialjs.org/missionstatement>. Cited: Apr. 26, 2018

Jacobson, S.; Marino, J.; Gutsche Jr., R. The digital animation of literary journalism. Journalism, v.17, n.4, p.527-546, 2016. http:// dx.doi.org/10.1177\%2F14648 84914568079

Jeanne-Perrier, V. Média imprimé et média informatisé: le leurre de la complémentarité. Communication et Langages, n.129, p.49-63, 2001.

Larrondo, A. La metamorfosis del reportaje en el ciberperiodismo: concepto y caracterización de un nuevo modelo narrativo. Comunicación y Sociedad, v.22, n.2, p.59-88, 2009.

Larssen, K.; Hornmoen, H. The Literary Journalist as fellow human being. Literary Journalism Studies, v.5, n.1, p.81-96, 2013.

Lassila-Merisalo, M. Story First: Publishing narrative long-form journalism in digital environments. Journal of Magazine \& New Media Research, v.15, n.2, p.1-15, 2014.

Le Masurier, M. What is Slow Journalism? Journalism Practice, v.9, v.2, p.138-152, 2015. http://dx.doi.org/10.1080/17512786. 2014.916471

Le Masurier, M. Slow Journalism. Journalism Practice, v.10, v.4, p.439-447, 2016. http://dx.doi.org/10.1080/17512786.2016.1 139902

López Pan, F. Periodismo literario: entre la literatura constitutivo y la condicional. Ámbitos, n.19, p.47-68, 2010.

Marsh, C. Deeper than the fictional model. Journalism Studies, v.11, n.3, p.295-310, 2010.

Martinez, M. Literary Journalism: Conceptual review, history and new perspectives. Intercom: Revista Brasileira de Ciências da Comunicação, v.40, n.3, p.21-37, 2017. http://dx.doi. org/10.1590/1809-5844201732

Masip, P. et al. Investigación internacional sobre ciberperiodismo: hipertexto, interactividad, multimedia y convergencia. El Profesional de la Información, v.19, n.6, p.568-576, 2010. 
McKay, J. Reportage in the U.K.: A hidden genre? In: Bak, J.S.; Reynolds, B. (Ed.). Literary Journalism across the globe. Amherst and Boston: University of Massachusetts Press, 2011.

Mejía, E. Albert Chillón enseña periodismo en Barranquilla. La Cháchara. Disponible en: <http://lachachara.org/2017/03/ albert-chillon-ensena-periodismo-en-barranquilla/>. Acceso en: 26 abr. 2018.

Micó, J.L. Periodisme a la Xarxa. Vic: Eumo Editorial, 2006.

Neveu, E. On not going too fast with slow journalism. Journalism Practice, v.10, n.4, p.448-460, 2016. http://dx.doi.org/10.1080/1 7512786.2015 .1114897

Neveu, E. Revisiting narrative journalism as one of the futures of journalism. Journalism Studies, v.15, n.5, p.533-542, 2014. http:// dx.doi.org/10.1080/1461670X.2014.885683

Palau, D. Literary Journalism: Bases, production and writing strategies. In: Peña Acuña, B.; Jover López, J.J. (Ed.). Periodismo Especializado. Madrid: Asociación Cultural y Científica Iberoamericana, 2017. p.67-96.

Paulussen, S. Online news production in Flanders: How flemish online journalists perceive and explore the internet's potential. Journal of Computer-Mediated Communication, v.9, n.4, 2004. http://dx.doi.org/10.1111/j.1083-6101.2004.tb00300

Pavlik, J. Journalism and new media. Nova York: Columbia University Press, 2001.

Pereira Lima, E. A century of nonfiction solitude: A survey of Brazilian literary journalism. In: Bak, J.S.; Reynolds, B. (Ed.). Literary Journalism across the globe. Boston: University of Massachusetts Press, 2011.

Puerta Molina, A.A. La crónica latinoamericana actual: Io maravilloso real: análisis del periodismo narrativo de Alberto Salcedo Ramos. 2016. 530f. Tesis (Doctorado) - Universidad Autónoma de Madrid, Madrid, 2016. Disponible en: <https:// repositorio.uam.es/handle/10486/673229>. Acceso en: 9 oct. 2017.

Quandt, T. et al. Online Journalists in Germany 2002: The first representative survey on German online Journalists. In:
Association for Education in Journalism and Mass Communication 2003 Convention. Kansas: AEJMC, 2003.

Rodríguez, J.M.; Albalad, J.M. El periodismo narrativo en la era de internet: las miradas de Orsai, Panenka, Anfibia, FronteraDy Jot Down. In: Angulo, M. (Ed.). Crónica y mirada. Madrid: Libros del K.O., 2013. p.85-121.

Rost, A. La interactividad en el periódico digital. 2006. 473f. Tesis (Doctorado) - Universitat Autònoma de Barcelona, Barcelona, 2006. Disponible en: <http://www.tdx.cat/handle/10803/4189>. Acceso en: 11 oct. 2017.

Salvatierra, M.C. Notas para la investigación del periodismo literario en publicaciones digitales argentinas. In: Jornadas de Nacionales de Investigación en Comunicación, 11., 2017, San Juan. Actas... San Juan: RedCom, 2017.

Singer, J. Changes and consistencies: Newspaper journalists contemplate online future. Newspaper Research Journal, v.18, n.1-2, p.2-18, 1997. http://dx.doi.org/10.1177/073953299701800101

Sims, N. Los periodistas literarios o el arte del reportaje personal. Bogotá: El Áncora Editores, 1996.

Soares, I. Literary Journalism's magnetic pull: Britain's "new" Journalism and the Portuguese at the Fin-de-Siècle. In: Bak, J.S.; Reynolds, B. (Ed.). Literary Journalism across the globe. Boston: University of Massachusetts Press, 2011.

Van Krieken, K.; Sanders, J. Framing narrative journalism as a new genre: A case of study of the Netherlands. Journalism, v.18, n.10, p.1364-1380, 2017

Vivaldi, G. Curso de redacción: teoría y práctica de la composición y del estilo. Madrid: Paraninfo, 1999.

Wolfe, T. El Nuevo Periodismo. Barcelona: Anagrama, 1973.

Zdovc, S. Zeljko Kozinc, the subversive reporter: Literary Journalism in Slovenia. In: Bak, J.S.; Reynolds, B. (Ed.). Literary Journalism across the globe. Amherst and Boston: University of Massachusetts Press, 2011. 